\title{
Le groupe prototype de Castet et ses perspectives d'avenir
}

\section{The Castet prototype unit and its future prospects}

\author{
PAR II. CHAMAYOC \\ JWGENUER EN GHEF A LA S.N.C.F. \\ CHEF DU SFRVICE DE L'ÉNERGIE ÉLECTRIQUE
}

Cet exposé comportait trois parties :

- Les idées directrices qui ont conduit à

l'étude et à la réalisation de ce prototype.

- Une description succincte de l'installation

de Castet et plus particulièrement des groupes.

- Enfin, l'exposé des résultats obtenus et des considérations sur l'avenir qui peut être réservé à ce type de groupe.

Les idées directrices sont de plusieurs ordres :

- Recherche sur les usines marémotrices;

- Améliorations techniques apportées depuis de nombreuses années par les constructeurs dans la construction des groupes, leur encombrement et leur puissance massique;

- Recherche d'économies sur les auxiliaires, au sens général, qui accompagnent dans une installation la partie active, c'est-à-dire le groupe turbine-alternateur. Le type de groupe en question permet notamment de supprimer le batiment d'usine;

.... Entin, meilleure liaison entre le turbinier et l'electricien obtenup par l'existence d'un groupe monobloc.

l.es deux premières parties ont été déjà développées dans plusieurs articles et notamment dans la Houille Blanche, $n^{\circ}$ de mars-avril 1954. Nous renvoyons le lecteur $\dot{a}$ cet article et nous nous bornons à reproduire les parties essenlielles de l'exposé traitant des résultats obtenus et des perspectives d'abenir du groupe.

14. Chamayou donne d'abord des renseignements sur les caractíristiques de ce groupe et les mises au point qui ont été nécessaires. Par rapport $\dot{a}$ une installation classique, une économie globale de $1 / 3$ a pu être réalisée. Cette économie n'est d'ailleurs pas la limite puisque des dispositions encore plus économiques pourront être et sont déjà apportées sur des groupes dérivant du mème type.

Quant aux perspectives d'avenir elles intéressent d'abord l'équipement des basses chules, l'utilisation des groupes du type Castet permettant des économies sensibles de génie cinil dans la rélisation des usines-barrages. Il est donc possible que dans certains cas les usines de ce type deviennent plus économiques que des usines du type a canal de dérivation.

Les autres applications possibles se trouvent dans les usines marémotrices et enfin dans les mirrocentrales.
This paper is made up of three parts:

- The motivating ideas which have led to the study and realization of this prototype.

- A brief description of the Castet installation and some detail about the wnits.

- Finally, the results obtained, and considerations of the future that can be expected for this type of unit.

There were several motinating ideas:

- Research for tida! power stations;

- Technical improvements developed oner " number of years by manufacturers in the construction of the units, the space they occupy and their power to weight ratio;

- General research of economies on the auxiliaries which accompany the active part of the installation i.e. accompany the turbinegenerator unit. The tlppe of unit in question enables the power station building to be obviated;

- Finally, the best cooperation between the turbine and electrical gear manufacturers obtained by the existence of a monobloc unit. The first two parts have already been treated in several articles, in particular la Honills Blanche of March-April 195. We refer the reader to this article and reproduce here only the parts essential to the treatment of the results obtained, and the future prospects of the unit.

Mr. Chamayou first of all gives some informittion on the characteristics of the unit and the developments which have been necessary. In comparison with a classical nnit an overall economy of $1 / 3$ has been realized. This economy is not the maximum that can be attained as even more economical arrangements can be and have already deneloped for similar installations.

As for the future possibilites, they mainly concern lour head equipment, the use of units such as Castet allows appreciable economies in the civil engineering work for the dam and power station. It is thas possible that powir stations of this type will sometimes be more eronomical than by-pass canal type stations.

The other applications possible are in tidal pouer stations and in midget power stations. 


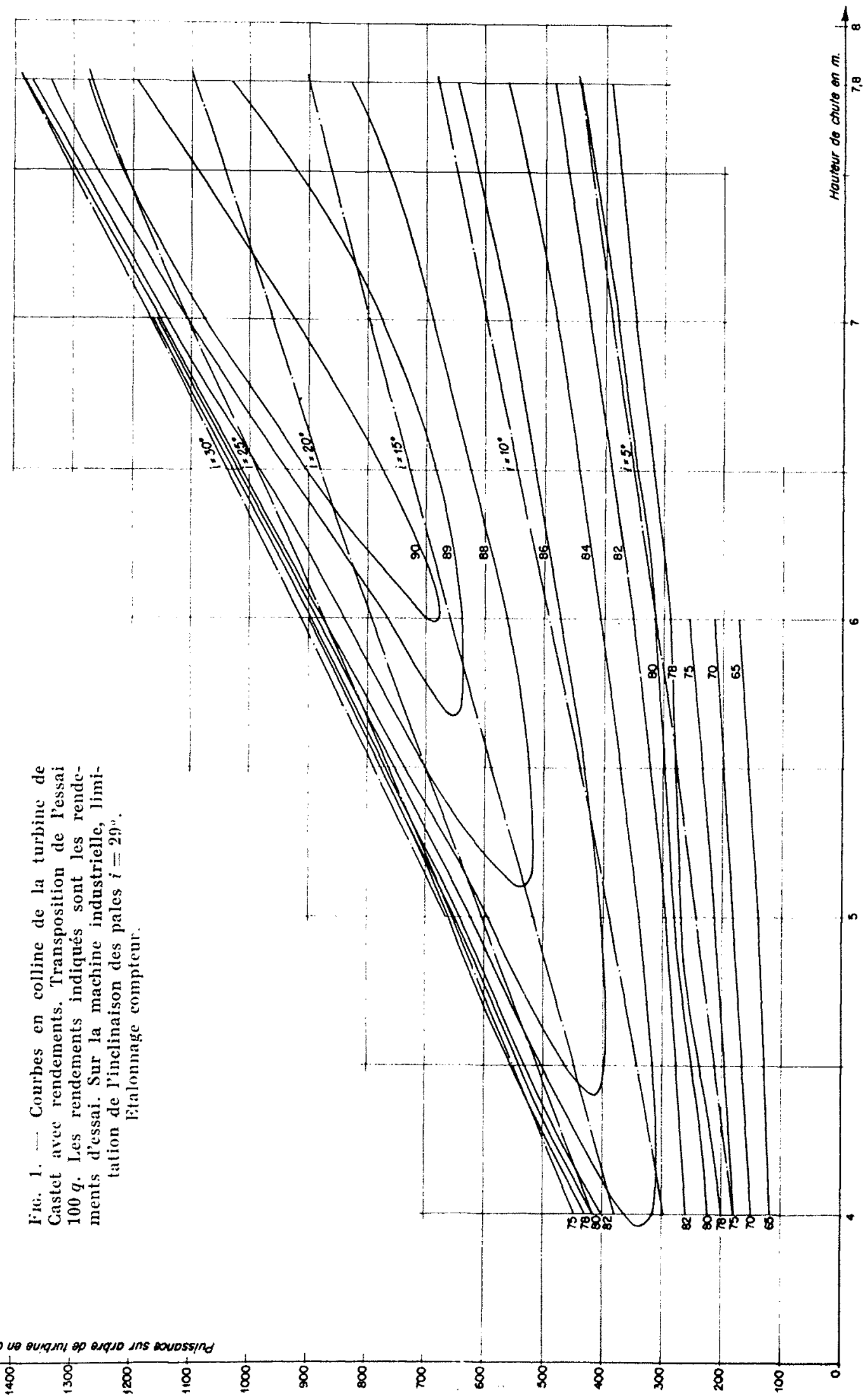




\section{Résultats obtenus. - Mise au point}

Comme dans toute réalisation nouvelle, on pouvait s'attendre à quelques imperfections et à quelques difficultés, même si les idées de base étaient particulièrement saines et séduisantes. En fait, les tentatives précédentes avaient échoué sur des détails technologiques.

Or, Ja mise au point des parties importantes et sur lesquelles on pouvait avoir, à priori, des inquiétudes a été extrêmement rapide. Seuls, quelques détails ont retardé la mise en service industrielle du groupe; en particulier, le problème des joints huile-eau a nécessité un certain nombre d'essais en usine el sur place. Les joints actuels donnent loute satisfaction. Ils ont une usure inappréciable et les pertes d'huile admises et inévitables sont fort acceptables : 1 litre environ par jour, représentant une dépense d'une centaine de francs.

On peut, dès à présent, affirmer que cette technique est satisfaisante et passer à l'étape suivante qui consiste à perfectionner ce qui existe déjà.

Nous rappelons que le but que nous recherchions nous-mêmes étail la réduction des dépenses de génie civil.

Dans l'installation, la part du groupe turbinealternateur représente $53 \%$ du total des dépenses contre $35 \%$ dans une installation classique.

En supposant que les groupes vaillent le même prix dans la solution classique que pour Castet, ce qui ne sera pas le cas ultérieurement puisque le groupe de Castet est incontestablement plus léger qu'un groupe classique de mème puissance, nous trouvons qu'une économie g]obale de $34 \%$ a été réalisée. Certes, une partie de cette économie est indépendante du type de groupe, mais nous insistons sur le fait que l'on peut aller encore plus loin et que les essais faits sur ces prototypes montrent que le maximum d'économie n'est pas encore atteint. Nous verrons plus loin que de nowvelles dispositions plus écomiques sont dès à présent possibles.

Il est intéressant de regarder quelle est effectivement l'origine des gains ainsi réalisés et à porler à l'actif du groupe *, Castet »:

Il n'y a pas de bàche spirale. Les fouilles ont été réduites au minimum, il n'y a même pas de fouilles nécessitées par le sroupe lui-même. celles du barrage suffisent. Il n'y a plus de gaines de circulation d'air froid et d'air chaud en circuit ouvert ou en circuit fermé.

La place du groupe dans le béton du barrage est représentée par un simple trou de forme conique. Il $y$ a donc simplification des formes.

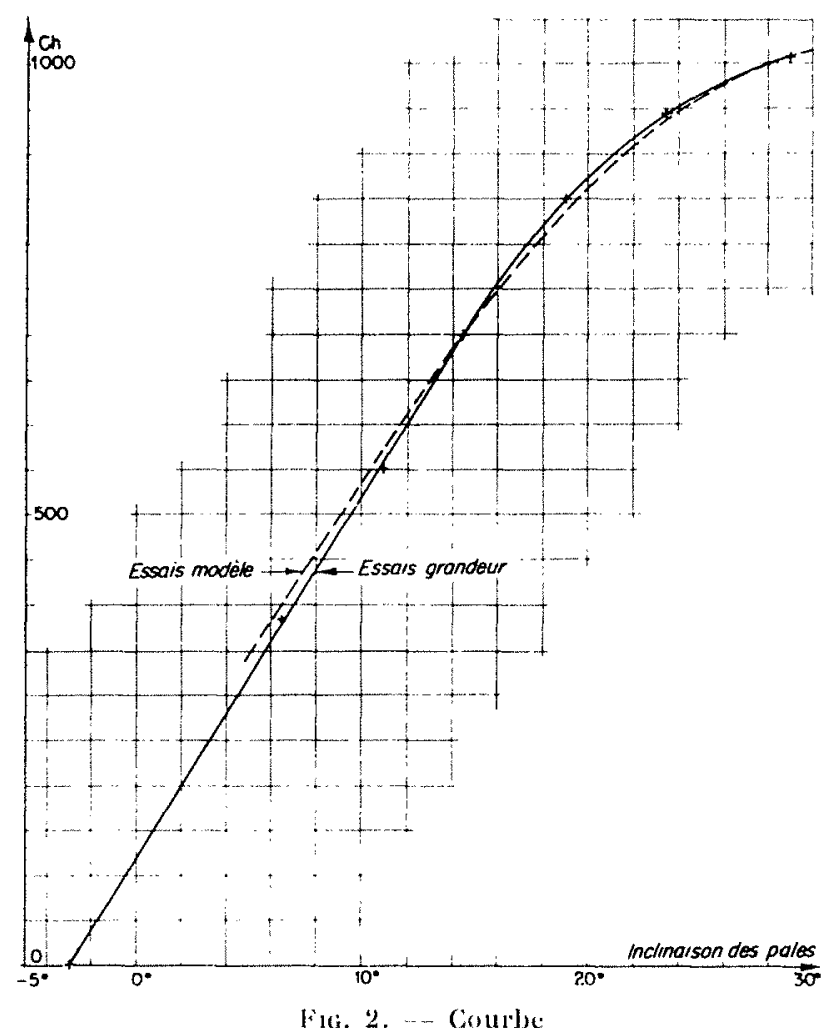

Puissince Ouverture: $\mathrm{H}_{n}=6,40 \mathrm{~m}$

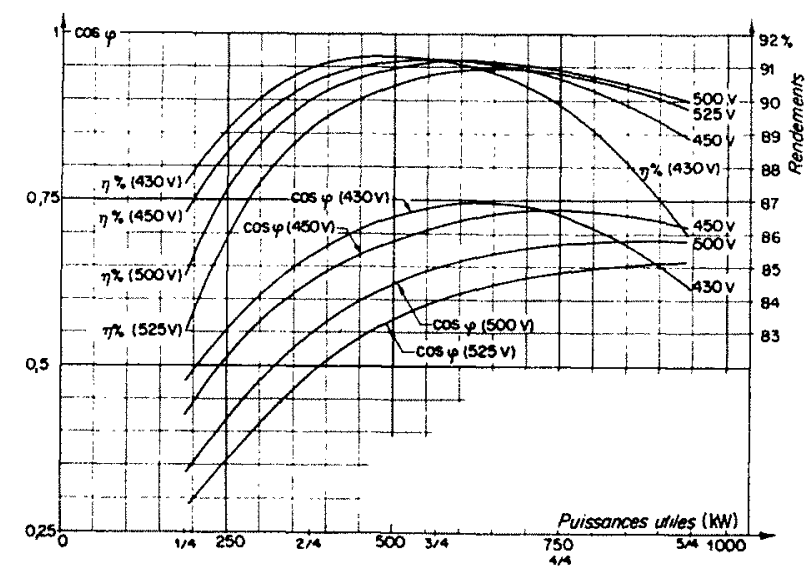

Fig. 3. - Génératrice asynchrone $750 \mathrm{k} \mathrm{kW}-254 \mathrm{t} / \mathrm{mm}$.

Courbes de rendements et facteurs de puissance en fouction de la charge pour differentes valeurs de la lension.

Obs. : Les rendements comprennenl les pertes supplémentaires évaluées a $10 \%$ des pertes joule siator.

La réfrigration a été simplifiée. Il n'y a pas de protection contre l'incendie el, bien entendu. il n'y a plus de bâtimenl d'usine.

\section{Rendements}

La figure 1 donne les courbes en colline résullant des essais sur modile. 
Nous n'avons pas encore fait d'essais complets de rendement, bien que toutes les mesures aient été prises pour pouvoir les exécuter. De tels essais comportent une marge d'incertitude pour tenir compte de l'imprécision des mesures industrielles. Nous avons estimé que les résultats les plus précis étaient ceux qui étaient donnés par les essais sur modèle en liboratoire.

Nous avons seulement relevé la courbe puissance-ouverture. La concordance avec la courbe théorique est très satisfaisante (fig. 2).

Ci-contre également les courbes de rendement et du facteur de puissance de la génératrice ilsynchrone (fig. 3).

Enfin, signalons qu'en service la température de l'huile du groupe n'a jamais dépassé $40^{\circ} \mathrm{C}$. (Il est vrai que la température de l'eau reste de lordre de $10^{\circ}$.)

\section{Améliorations consécutives aux essais}

Dès à présent, il est certain que diverses modifications seraient apportées aux groupes, s'ils étaient à refaire, compte tenu de l'expérience acquise.

Sans trop insister sur ce qui constitue peutètre des secrets de fabrication, mais que lon peut mentionner puisque des groupes en commande en seront munis, indiquons que lat gaine qui relie le groupe à l'extérieur serait très diminuée par l'inclusion du régleur à l'intérieur du bloc. La liaison rigide représentéc par la gaine serait remplacéc par de simples câbles souples, ce qui faciliterait encore le montage et augmenterait l'autonomie du groupe par rapport à son appareillage.

Une pompe de circulation d'huile placée par prudence dans le bloc ne s'avère pas indispensable.

On pourrait rendre démontables les parties sroupes-distributeur pour faciliter les transports.

Pour des groupes de plus faible puissance, on a déjà réalisé à la Maignannerie (89) ch sous $2,25 \mathrm{~m}$ ) une installation avee siphon qui permettra la suppression de la vanne de garde, l'arrêt du groupe se faisant par admission d'air en tête du siphon.

On peut concevoir, quand il y a de nombreux groupes, que ceux-ci soient à pales fixes. Le déhit serait réglé par le distributeur et le groupe pourrait même fonctionner par tout ou rien, les directrices étant alors fusionnées avec la vanne d'entrée.

\section{Perspectives d'avenir}

\section{EXTRAPOLATION}

On peut concevoir les variations des dimensions dans le sens de l'augmentation et dans celui de la réduction.

Les résultats très satisfaisants obtenus pour le refroidissement montrent que l'on pourrait aisément augmenter la puissance de l'alternateur.

Le diamètre de la roue, de $1,650 \mathrm{~m}$, pourrait, sans aucune difficulté el sans aucun risque supplémentaire, ètre porté à $2,50 \mathrm{~m}$ ou $2,60 \mathrm{~m}$. En fait on est surtout limité par des possibilités pratiques de transport. Mais le poids du groupe de Castet $(16 \mathrm{t})$ peut ètre dépassé, et nous avons vu qu'il est facile de le fractionner : le démontage et le remontage se sont révélés tellement firciles et rapides cqu'à Castet, pour effectuer certaines visites ef modifications pendant la période des essais et mises au point, il a suffi de poser le groupe dans la cour de l'usine sur un camarteau, et de procéder sur place aux démontages et remontages. Tout au plus avait-on prévu de mettre en place une bâche si le temps avail menacé.

Avec le système actuel d'alternateur dans l'huile, on peut dès à présent affirmer qu'il n'y a aucune difficulté à pousser la puissance de groupes semblables à ceux de Castet jusqu'à 5.000 ch pour une douzaine de metres de chute.

Rien ne prouve d'ailleurs qu'il ne soit pas possible d'aller au-delà, mais il sera peut-être nécessaire pour des puissances plus grandes d'adopler soit l'air comprimé, soit éventuellement l'hydrogène. A priori, on peut penser que l'utilisation de l'air comprimé à la pression de $5 \mathrm{~kg}$ rétablirait, en ce qui concerne l'évacuation des pertes, les conditions de fonctionnement dans l'huile.

Quant a la technique des groupes tournant dans l'hydrogène, quoique connue depuis très longtemps, clle tend maintenant à se généraliser en faisant même circuler le fluide à l'intéricur des conducteurs.

Il est rrai que l'on peul avoir quelque répugnance à adopter des groupes asynchrones, pour des puissances élevées à cause de leur mauvais facteur de puissance et de leur manque d'autonomie. Pour Castet, ces inconvénients sont mineurs, le $\cos$ i n'est d'ailleurs pas spécialement mauvais d'autant que les électriciens particulièrement prudents, ont, au dernier moment, ausmenté légèrement l'entrefer. De toutes façons, il est toujours possible d'améliorer ce facteur de puissance par l'installation de batteries de 
condensateurs. On peut egalement concevoir dans une installation comportant de nombreux groupes, linstallation de 1 on: 2 compensaleurs synchrones utilisés pour l'ensemble de la centrale, permettant aussi de conserver pour les groupes les avantages de la génélatrice asynchrone : poids, simplicite, couplage fait mème ì l'arrèt, suppression de l'excitatrice, etc.. En bref, les avantages de simplicité et de prix l'emportent pour les faibles puissances et les petites vitesses. Il n'en serait sans doute pas de nême pour les fortes puissances.

On pourrait encore inroquer l'insulfisance du PD2 pour limiter l'extension de la puissance des groupes de Castet. Mais ce n'est que sur le plan général que la question se poserait, s'il $y$ avail une très forte proportion de groupe de ce type.

On peut done sans optimisme exagérẻ envi-, sager l'augmentation de la puissance de ces groupes dans de grandes proportions et E.D.F. a déjà passé des commandes dans le but de s'assurer des possibilités d'extrapolation.

Tout ceci explique la révolution, à laquelle je faisais déjà allusion au début de cet exposé, sur la façon d'envisager l'équipement de rivières importantes et même de fleuves.

On sait que les usines basse chule jeuvent se classer en deux grandes catégories : usines a canal de dérivation et usines barrage. Lal première technique est assez souvent employée en France; au contraire, en Amérique, sur des cours d'eau importants, les usines sont généralement des usines barrage, e'est-à-dire que le barrage arec ses ouvrages annexes, l'usine et éventuellement l'écluse, forment un seul bloc.

Pour une puissance installée déterminée, l'emploi de groupes de moins en moins nombreux mais de plus en plus puissants avait en sa faveur l'encombrement global moindre, et une économie sur l'appareillage électrique, mais cette tendance au gigantisme, pour l'hydraulique tout au moins, esl nellement en regression.

De plus, si un groupe classique, de puissanes $P$, est, pour la dimension commandée par le profil en traver's du cours d'cau, moins encombrant que deux groupes classiques de puissance $\mathrm{P} / 2$ et, a fortiori, de quatre groupes de puissance $\mathrm{P} / 4$; pour des groupes du lype Castel, l'augmentation de l'encombrement est beaucoup plus faible. Et ceux-ci pourraient mème être surmontes de vannes de erue, ils n'inlervicudraien! donc pas dans le rétrécissement de la section disponible pour le passage da flot.

Compte lenu des inconvénients el des dépenses qui résultent de la construction de longs et importants canaux. on pressent que la comparaisón entre les deux types d'usines peut êtr' reprise à la lumirre des réalisations Castet, et si les expériences entreprises par E.D.F. contirment les résultats de Castet, il n'est pas du tout certain que, dans un avenir plus ou moins loinlain, l'avantage technique et financier ne revienne pas, dans certains cas, aux usines barrage i groupes nombreux du type immergé.

\section{USINES MAREMOTRICES}

Il est bon de rappeler que ce sont les préoceupations des usines marémotrices qui, pour certains ingénieurs, ont été à l'origine des recherches qui ont conduit ä ce nouveau type de groupes. Il est certain que le groupe-type Castet se prêterait particulièrement bien à une telle utilisation. Il faut également noter que de tels groupes peuvent aisément fonctionner de quatre façons, c'est-à-dire en turbine dans les deux sens, en pompe dans les deux sens.

Or, pour Castet qui n'avait pourtant pas été étudié spécialement pour cela, on arrive déjà à des rendements qui sont très honorables aussi bien en turbine qu'en pompe. Les groupes de Cambeyrac sont concus pour permettre cette quadruple utilisation, et pour mesurer les rendemenls correspondants.

\section{Microcentrales}

Depuis longtemps déjà l'attention s'est portée sur les microcentrales. On sait qu'il est convenu d'appeler ainsi des installations de faible puissance dont les groupes sont tries simples, construits en série, donc bon marché, et pourraient être placés sans grands frais sur des cours d'eau modestes.

Ces installations à l'échelle des particuliers, industriels, propriétaires ruraux pourraient totaliser pour l'ensemble du pays une puissance el une production de l'ordre de celle de plusieurs grands ensembles. Débitant leur courant sur le lieu même de leur ulilisation, elles échapperaient aux, sujétions et aux pertes inhérentes aux reseaux de transport ef de distribution, sur les dépenses desquelles elles permethaient do faire des économies imporlantes. D'un coùl moderé, elles ne poseraient aucun problène de financement a l'échelle nationale ${ }^{*}$ ).

\footnotetext{
(") Rien de llouveau suus le soleil : se basant sur un projet de capitation de Vauban. 1694, on a pu estimer qu'il $y$ avait à ce moment, en France, 85.000 moulins à eau (non compris les moulins à vent) dont 70.000 pour le blé, 15.000 pour d'autres usages (foulons, battoirs ì chanvre, moulins it papier, forges et martinets et fonderies), dont 30.000 dans le scul ressort du Châtelet de laris.
} 
Cette idée de construire des groupes très simples suivant une gamme limilée de types en fonction du débit et de la hauteur de chute, a déja séduit plusieurs constructeurs, mais le groupe, type Castet, se prête particulièrement bien ì cette construction. Le groupe de la Maignannerie avec siphon, dont j’ai déja parlé, en est une application réussie.

\section{Conclusion}

J'ai, au cours de cel exposé, montré que la realisation du groupe de Castet, résultant des efrorts combines des hureatux d'études de Xevrpic et d'Alsthom, d'ingénieurs éminents, iols M. Guimbal, ef entin de nous-mêmes, auxquels il est également justice d'associer l'entreprise du Génie Civil Société des Travaux de Nonlagne et Sainrapt et Brice, a donné les résultats que l'on escomptait à priori, el est dès à présent susceptible de développements inléressants.

Il est assez satisfaisant, pour l'amour-propre national, que ces groupes, conçus el construits chez nous, soient 100 of francais.

\section{DISCUSSION}

Président : M. G+ReL

M. Ferny pense que la défance manifeste par M. Снаsiayoe a l'egard des mesures in sita n'est pas aussi générale que pourrait le laisser croire son allusion à ce sujet et s'applique exclusivement an cas des basses chutes. Cest cu effet lo eas le plus difficile; mais même alors, en particulier lorsque la question a été étudiée dès la construction de l'usine, il estime que l'erreur de mesure peut ctre réduite a une valeur inférieure à celle qui résulte de la simple extrapolation des résultals d'essais sur modèles reduits. Il rappelle en outre, que, lorsqu'il s'agit de turbines Kaplan, les essais in situ, même cntachés d'erreurs systématiques, permettenl de délerminer la conjugaison optimum entre les pales ef l'onverture du distributeur, ce qui entraine souvent une amélioration appréciable du rendement par rapport à la conjugaison fixé par le constructeur, d'apres les données theriques et les cssais sur modèle.

M. Chanayoz est daceord sur la remarque de M. Frany d'autant plus que, dans le groupe étudic, les dispositions ont ete prises pour faire des mesures in situ.

M. Cabanes souhaite que la prépondérnace du materiet uoble, preconise par M. Cnnmaror pour les ouvages d'amenagement hydrauliqua, puisse etre etendue au domaine electrotechnigue : lignes de transport, transformateurs, interrupteurs, alternateurs : des résultats très intéressants ont dejà été obtenus par son serviee dans le domaine du transporl de l'encrgite (lignes et postes). II estime, d'autre part, que s'il est souhaitable pour tout ingénieur de conserver un fond d'unirersalité, la spécialisation permet, toutefois, de faire progresser plus rapidement la technique. I! pense aussi que pour des groupes plus puissants que coux de Castets le poids par kilowaft de puissance doit pouvoir itre encore plus reduit.

21. Berfrs.s indique qu'il $y$ a toutefois une limite infi. rienve des dimensions du groupe pour une puissance donnée: cette limite est imposée par les dimensions ne nerietur du circuil inductif, par les calories perdues qui. malgré leur refroilissemenl facile, diminuent d'autant le rendement of par la surepaisseur des enveloppes qui doivent etre prérues en fonction du diamelre pour résisw ter a la pression hydraulique du milieu; d'autre part, si la simplicite de la machine asynchrone élimine certains impedimenta et diminue le prix, tout an moins pour les machines de faible vitesse dont le circuit inducteur imposerait un dimensionnement exagée, il ne faut pas ou. blier que l'influence du facteur de puissance réduit très scnsiblement son intérêt récl.

M. Hexpy demande si la situation horizontale on pres que horizontale de ces groupes ne favorise pas l'apparition de la cavitation dans la partic haute de la machine, ce qui crécait, a l'emballement, unc dissometrie, unc espèce de balourd qui rendrait le phenomène de l'emhallement, toujours désagréable, encore plus diffeile, surtout pour les grandes machines.

II. Chamayou est daccord sur le risque d'emballement dissymetrique en cas de caritation, signale par M. HeNny, mais indique qu'll n'y a pas eu, a Castet, de tendance a la cavitation.

M. Ie Président précise qu: la cavilation est theoriquement plus dangereuse à la partic supérieure de la roue d'une turbine at axe horizontal, mais eomme cetle disposition supprime toute la partie du tuya d'aspiration qui, dans un groupe a axe vertical exigernit un enfoncement considérable, la hauteur totale d'un groupe à axe horizontal est, tout comple fait, inférieure de 0.7 a une fois le diamètre d'une roue a axe vertical présentant, dans son plan moyen, le même danger de cavitation maximum. Quant a la dissymétric de leffet métenique de la cavitation, a supposer que celle-i soit esalement dissymétrique au point de rue hydratique, M. le Présidenl ne croit pas que les groupes a axe horizonlal n'y soient point sujets aussi, ni que exs dissymótries meraniques soient plus faciles a supporter sur des paliers verticaux quavec des paliers horizontaux. Le risque signale par M. Hesnx s'aceroit evidemmenl aree la puissanes des spoupes ef c'est une des misons pour lesquelles il faut multiplier ces derniers, ee qui procute, d'alleturs. nue importante éenomie de génie civil.

Parmi les divers facteur's d'économic indiqués par M. Chamayor, M. Langlors a nolé. en partienlier, celui des économies de personnel d'exploitation dues is la possibilité de fonetionnement automatique de groupes de ce senre.

Cette question est d'alleurs bien connue dPE.D.F, puisque l'une des premieres preoccupations du Service de la froduction Hydraulique, lors de la mationalisation. at ete préciscment la mise en application do celle politique de réduction des effectifs, qui a conduit notamment is la mise sur pied d'un programme de semi-atitomalisalion it (les degres divers de nombreuses installations existante et, pour certaines d'entre elles, if lew automatisation totale ou a leur télecommande.

11 est done normal de penser que pour un entemble to miero-centrales atilisant des groupes de lordre de grandeur de celui de Castet, la télecommande permettrait de n'avoir notamment qu'un personnel le survellance rela. tivement réduit placé au centre d'explotiation de l'ensemble des centrales groupées. Par contre, pour des trosses centrales comportant de nombreux groupes, la question devrait être regardee de plus près car la complication des circuits et des chaines de relais d'automatisme conduit pratiquement it limiter it deux ou trois le nom 
bre des troupes thes centrales entiofement automatiques ou teléconmandés, encore que dans ce dernier cas la listance do téléeommande constitue un paramètre supplémentaire de rentabilite.

M. Cramaxou précise que la question de lautomatisme et de la télécommande ne sont liés au probleme du sroupe prototype de Castet que dans la mesure où ce probleme suppose la multiplication des groupes ponr etre étendu aux grosses centrales, mis il n'est quand mème pas concevable qu'une telle centrale soit laissex sans aucune surveillanee humaine.

M. Bezlros s'associo a la dernière remarque de M. C.uA sarov et pense qu'n dehors des petites centrales a machines asynchrones simples interconnectécs sur un réseau, lautomalisme ne peut che ponsse trés loin.

11. Canaxis souligne que l'interêt do lautomalisme do ce genre de centrie pen bue acou par le fait que le service demande a chacun des groupes peut se simplifter enormement (marche par tout ou rien), co qui simplitie considerablement lautomatisme.

Afn de lever toul ambirulte, M. Lavarous précise qu'il faut éviter de confondie la notion de semi-automatisalion avec l'automatisation totale on la lelecommande.

En effet, dans le premier cas, les dispositions a prendre fout en ne conduisant pas a une grande complication des circuils clectriques, permettent cependant une réduction jusqu'à 2 ou mème à 1 surveillant par quart.

Par contre, lantomatisation lotale et la telecommande. solutions plus complexes at plus couteuses, permettent la suppression du personnel do surveillane en permanence - sauf peut-itte un gatien dans quelques cas particuliers - mais impliquent par contre des visitos périodiques dentretien par un persomel specialisé. An surplus, ces solutions ne peurent gucre s'envisager qu'aveo un nombre de groupes relativement réduil.

Répondant a une question de M. Beav sur la rentabilité des barrages existant sur les cours deau frangais équipes avec les groupes tubulaires, M. Chamayou explique qu'il existe un seuil au-dessous duquel tout aménagement cesse j'etre rentable, mais que l'equipement propose, du fail de son economie propre et de sa possibilité de construction en serie, aumit pour effet d'abaissu le prix de revient de nombreuses installations actuellement reputées non rentables, qui passeraient ainsi dans la categone des installations renlables.

M. Martinet attire lattention sur lécueil qui consisterait a equiper celles de ees petites centrales qui seraient suseptibles d'êtro supprimés ultéricurement par suite de linstallation d'une grande centrale dans le mème bief, ainsi que cela c'est produit naguèro.

M. Remexienas montre que, pour les miero-centrales, si on arrive assez facilement a normaliser la machine et a reduire son prix, il reste tres difficile de normaliser lo génie civil dont la paut est souvent préponderante dans le coût de l'aménomemt. L'entretien des ourrages de génie civil des micro-centrales est plus coûteux par kilowatt produit que celui des usines puissantes cengravelaent des biefs, affoullements, etc...).

En ce qui coneerne les essais, M. Rembntenas est daecord avec M. Fenpy et ajoule que, si dans le cas d'une chute da $6,40 \mathrm{~m}$ la comparaison des courbes puissanceouverture de la machine modele et de la machine cu vraie sranden, est assez satisfaisante, comme on pouvait sy attendre, il serait intéressant, dans le cas général, de connatre lécart des rendements admis respectivement pour la turbinc modèle pour la machine réclle.

1. Le Pésident estime que la diffèrence de diamétres entre les 2 turbines ci-fessus étant fable, les differences de readement sont extremement fables. lorsqu'on passe du modèle a la rraie srandeur.

17. Chamsot revond qu'il a sarrifté par necessite économique les essais in sith qui somt relalivement couteux et sans gros inleret pour la S.N.C.F. mais que l'usine a élé prevue pour les fatre ef quelle est a la disposition d'Electriejte de France.

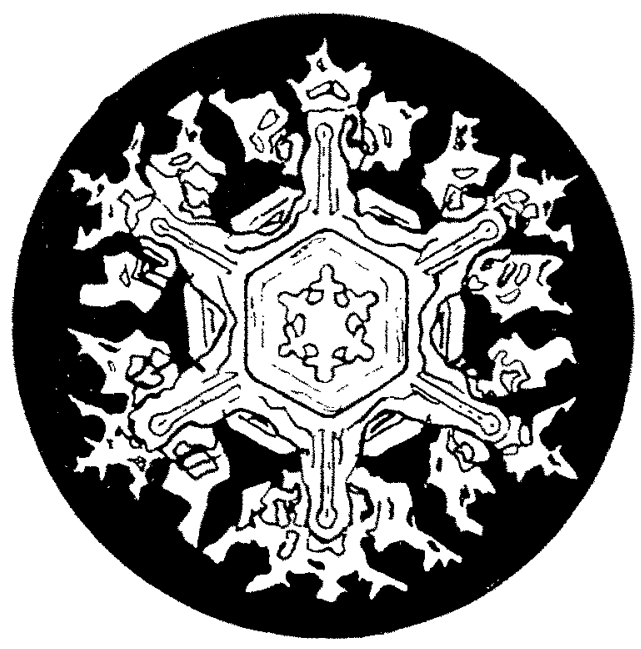

